\title{
Risperidone treatment for ADHD in children and adolescents with bipolar disorder
}

\author{
Joseph Biederman \\ Paul Hammerness \\ Robert Doyle \\ Gagan Joshi \\ Megan Aleardi \\ Eric Mick
}

Pediatric Psychopharmacology

Research Department, Massachusetts

General Hospital, Boston, MA, USA

Correspondence: Joseph Biederman

Warren 705, Massachusetts General

Hospital, Pediatric Psychopharmacology

Research, 55 Fruit St., Boston, MA. 02I I4,

USA

Tel +I 6177240956

Fax +| 6177261743

Email jbiederman@partners.org
Objective: Children and adolescents with bipolar disorder are also at high risk of having comorbid attention-deficit hyperactivity disorder (ADHD). The objective of this study was to estimate improvement in ADHD symptoms in children with bipolar disorder.

Methods: This was an open-label, study of risperidone monotherapy for the treatment of pediatric bipolar disorder. Thirty-one children and adolescents $4-15$ years of age ( $7.2 \pm 2.8$ years) of both sexes $(71 \%, \mathrm{~N}=22$ male) with pediatric bipolar disorder (YMRS score $=32.9 \pm 8.8$ ) and ADHD (ADHD-RS score $=37.9 \pm 8.9$ ) were included in these analyses.

Results: Improvement in ADHD symptoms was contingent on improvement in manic symptoms. Although both hyperactive/impulsive $(-7.5 \pm 5.5 .6, \mathrm{p}<0.05)$ and inattentive $(-6.8 \pm 5.0$, $\mathrm{p}<0.05)$ ADHD symptoms were significantly improved with risperidone, improvement was modest, and only $29 \%$ of subjects $(\mathrm{N}=6)$ showed a $30 \%$ reduction in ADHD rating scale scores and had a CGI-I $\leq 2$.

Conclusions: These results suggest that that treatment with risperidone is associated with tangible but generally modest improvement of symptoms of ADHD in children with bipolar disorder.

Keywords: ADHD, bipolar disorder, children, risperidone

\section{Introduction}

Childhood bipolar disorder (BPD) is among the most severely disabling psychiatric conditions affecting children (Faedda et al 1995; Geller and Luby 1997; Biederman et al 2000). It is associated with great severity of the illness including psychosis, mixed mania, and high rates of aggression and impairment (Strober et al 1995; Wozniak et al 1995; Geller et al 2002).

In a recent prospective, open-label, treatment trial (Biederman et al 2005b), we documented that treatment with risperidone was associated with clinically and statistically significant improvement in manic symptomatology indexed through the Young Mania Rating Scale (YMRS ) $(-14.4 \pm 10.6, \mathrm{p}<0.0001)$. Using predefined criteria for improvement, the response rate for manic symptoms was $70 \%$ (Biederman et al 2005b). However, since studies of children with bipolar disorder consistently document a very high degree of overlap between pediatric bipolar disorder and attentiondeficit hyperactivity disorder (ADHD) (Borchardt and Bernstein 1995; Geller et al 1995; West et al 1995; Wozniak et al 1995; Biederman et al 2000; Biederman et al 2004), whether treatment with risperidone is also effective in the treatment of ADHD symptomatology in children with bipolar disorder remains uncertain.

The available literature suggests a role for risperidone in the management of ADHD symptoms in the context of disruptive behavior disorders. In a randomized placebo-controlled clinical trial of children with disruptive behavior disorders and subaverage intelligence, Aman and colleagues (2004) showed that risperidone was associated with improvement in symptoms of hyperactivity, regardless of concomitant 
stimulant use. Likewise, in a single blind randomized trial of methylphenidate or risperidone treatment of ADHD children with moderate mental retardation (Correia Filho et al 2005), risperidone was effective as methylphenidate for ADHD symptoms. Eapen and colleagues (2005) examined a small sample of children with disruptive behavior disorders, ADHD and aggression and demonstrated a positive clinical response in 9 of 12 children treated. Yet, to the best of our knowledge no study has specifically addressed the impact of risperidone in the treatment of comorbid ADHD in children with bipolar disorder.

Whether treatment with risperidone is effective in the treatment of ADHD symptoms in children with bipolar disorder is of high clinical significance. Considering the high overlap between bipolar disorder and ADHD and the high morbidity associated with ADHD, addressing this disorder can greatly facilitate the management of children with bipolar disorder. Since traditional treatments for ADHD can exacerbate mania, the identification of alternative treatments to manage ADHD symptomatology in children with bipolar disorder devoid of a destabilizing potential are needed.

The objective of this study was to evaluate the effects of risperidone monotherapy on ADHD symptoms in a sample of children with bipolar disorder. To this end we conducted secondary analyses examining response to ADHD symptoms in a prospective open-label trial of risperidone utilizing systematic clinical assessments of ADHD severity and improvement. Based on the literature, we hypothesized that ADHD symptoms would improve in children with bipolar disorder and comorbid ADHD and that improvement in ADHD symptoms in bipolar children will only be observable in those who improved their symptoms of mania.

\section{Methods}

As previously reported (Biederman et al 2005a, 2005b), the study consisted of an 8-week, open-label treatment trial of risperidone monotherapy in children with bipolar disorder. All study procedures were reviewed and approved by our Institutional Review Board for Research on Human Subjects. All subjects' parents or guardians signed written informed consent forms and children ( $>7$ years of age) signed written assent forms.

Male or female subjects 4-17 years of age were included in the trial. Each subject met criteria for DSM-IV bipolar I disorder, or bipolar disorder not otherwise specified (NOS) and were currently displaying manic or mixed symptoms (with or without psychotic features) based on clinical assessment by a board-certified child and adolescent psychiatrist.
The DSM-IV requires subjects met criterion A for a period of extreme and persistently elevated, expansive or irritable mood lasting at least one week, plus criterion B, manifested by three (four if the mood is irritable only) of seven symptoms during the period of mood disturbance. Also recorded was the onset of the first episode, the number of episodes and the total duration of illness. BPD II disorder was defined according to the DSM-IV as hypomania (an abnormal mood lasting at least 4 days), and BPD NOS was defined as a severe manic mood disturbance that did not meet either time criteria (at least 4 days) or had fewer elements in criterion B (only required 2 items for elation and 3 for irritability).

In order to assess the lifetime and current rates of psychiatric comorbidity structured diagnostic interview Kiddie Schedule of Affective Disorders and Schizophrenia Epidemiological Version (KSADS) (Ambrosini 2000) were conducted with mothers and children over the age of 12 years. KSADS interviews were administered by highly trained and supervised non-clinician interviewers. All diagnoses were reviewed by a sign-off committee of experienced board certified child and adolescent psychiatrists chaired by the senior investigator (JB).

In addition, all children were clinically assessed for bipolar disorder and other psychiatric comorbidity by boardcertified or board-eligible child and adolescent psychiatrists. During this baseline evaluation the clinicians completed the Young Mania Rating Scale (YMRS) (Young et al 1978; Youngstrom et al 2002) as a measure of current symptom severity of mania. To be included in the study, subjects must have had a score of 15 or greater on the YMRS. ADHD severity was assessed at baseline, week 4 and endpoint with the clinician rated ADHD Rating Scale (ADHD-RS). To assess clinically significant severity and improvement relative to baseline, we used the NIMH Clinical Global Impression (CGI) severity (CGI-S), and improvement (CGI-I) scales (National Institute of Mental Health 1985) rated separately for symptoms of mania, depression, and ADHD. The score for the CGI-S ranges from 1 (normal, not at all ill) to 7 (among the most extremely ill patients). The score for the CGI-I ranges from 1 (very much improved) to 7 (very much worse). Subjects were considered ADHD responders a priori if they had at least a $30 \%$ reduction on the ADHD-RS and an ADHD CGI-I $\leq 2$ (much or very much improved).

We excluded subjects with any serious, unstable illness including hepatic, renal, gastroenterologic, respiratory, cardiovascular (including ischemic heart disease), endocrinologic, neurologic, immunologic, or hematologic disease. Subjects with DSM-IV substance (except nicotine 
or caffeine) dependence within past 6 months or who were judged to be at serious suicidal risk were also excluded from participation. No subjects were tapered off of their current antimanic medications for the purpose of enrolling in this study unless the current treatment was determined to be ineffective as indicated by continuing to meet entrance criteria (active symptoms of mania and a YMRS Score of $\geq 15$ ). Mood stabilizers, anticonvulsants, and other neuroleptic therapy, were not allowed during this study. Antidepressants were exclusionary to the study.

Risperidone was initiated at an open-label dose of 0.25 $\mathrm{mg}$ /day for children $\leq 12$ years and $0.5 \mathrm{mg}$ /day for older youth to be increased weekly according to response and tolerability to a maximum does of $2.0 \mathrm{mg} /$ day for $\leq 12$ and up to $4.0 \mathrm{mg} /$ day for older youth. Given the strong overlap of juvenile bipolar disorder with attention deficit hyperactivity disorder, the psychostimulants methylphenidate hydrochloride, dextroamphetamine sulfate and mixed amphetamine salts were allowed during the study if, in the clinician's judgment, it was in the best interest of the subjects to continue this treatment or if the subject did not wish to stop stimulant treatment and only if the subject had been on a stable dose for at least 30 days. If extrapyramidal symptoms occured, benztropine mesylate was allowed in doses of up to a maximum of $2 \mathrm{mg} /$ day. The use of the benzodiazepine lorazepam was permitted during the study in doses of $2 \mathrm{mg}$ or less per day. Nonpharmacological treatments such as individual, family, or group therapy were allowed if they were in place before the subject joined the study and if the therapy regimen remained the same throughout the study.
Safety was assessed at each visit using spontaneous reports of treatment-emergent adverse events, changes in vital signs and laboratory measures. Blood pressure and weight were recorded at each visit. Prolactin, glucose, and lipid levels were obtained at baseline and post treatment.

\section{Statistical analysis}

Generalized estimating equation models were used to analyze the repeated measures of the study as implemented in STATA. All analyses were intention to treat (ITT) with the last observation (LOC) carried forward for subjects that did not complete the entire study. Statistical significance was determined at $\mathrm{p}<0.05$.

\section{Results}

Although 59 children and adolescents were enrolled in this open-label treatment trial of risperidone monotherapy, only 36 had available ratings on the ADHD-RS. Five subjects who were receiving stimulant medications were excluded from further analyses. Thus, the final sample consisted of 31 children and adolescents $4-15$ years of age ( $7.2 \pm 2.8$ years) of both sexes $(71 \%, \mathrm{~N}=22$ male) with pediatric bipolar disorder (YMRS score $=32.9 \pm 8.8$ ) and ADHD (ADHD-RS score $=37.9 \pm 8.9)$. The mean dose of risperidone at endpoint was $1.47 \pm 0.67 \mathrm{mg} /$ day and $68 \%(\mathrm{~N}=21)$ were considered much or very much improved $(\leq 2)$ on the mania-specific CGI-I rating.

As shown in Table 1, ADHD symptom scores at baseline were slightly higher in the group that did not have an adequate antimanic response to risperidone. A statistically significant

Table I ADHD rating scale scores at baseline and endpoint in 31 bipolar youth

\begin{tabular}{|c|c|c|c|}
\hline & $\begin{array}{l}\text { Mania CGI-I } \leq \mathbf{2} \\
\mathbf{N}=\mathbf{2} I\end{array}$ & $\begin{array}{l}\text { Mania CGI-I > } 2 \\
\mathbf{N}=10\end{array}$ & \\
\hline & Mean \pm SD & Mean \pm SD & Statistic \\
\hline \multicolumn{4}{|c|}{ ADHD RS total } \\
\hline Baseline & $35.4 \pm 8.5$ & $43.3 \pm 7.3$ & $F(I, 29)-6.3, p=0.02$ \\
\hline Endpoint & $21.2 \pm 10.6^{*}$ & $39.0 \pm 7.8$ & $F(I, 29)-22.2, p=0.00 I$ \\
\hline Change & $-14.2 \pm 10.3$ & $-4.3 \pm 9.1$ & $F(I, 29)-6.8, p=0.02$ \\
\hline \multicolumn{4}{|c|}{ ADHD RS IA } \\
\hline Baseline & $17.8 \pm 4.4$ & $19.4 \pm 6.3$ & $F(I, 29)-0.6, p=0.9$ \\
\hline Endpoint & $\mathrm{II} . \mathrm{I} \pm 5 . \mathrm{I}^{*}$ & $17.4 \pm 5.8$ & $F(I, 29)-9.4, p=0.005$ \\
\hline Change & $-6.8 \pm 5.0$ & $-2.0 \pm 6.7$ & $F(I, 29)=5.3, p=0.03$ \\
\hline \multicolumn{4}{|c|}{ ADHD RS HI } \\
\hline Baseline & $17.6 \pm 5.8$ & $23.9 \pm 2.6$ & $F(1,29)-10.6, p=0.003$ \\
\hline Endpoint & $10.1 \pm 6.7^{*}$ & $21.6 \pm 4.7$ & $F(I, 29)-23.3, p<0.00 I$ \\
\hline Change & $-7.5 \pm 5.6$ & $-2.3 \pm 4.5$ & $F(I, 29)=6.4, p=0.02$ \\
\hline
\end{tabular}

Notes: ${ }^{*} \mathrm{p}<0.05$ within responder group from baseline to endpoint.

Abbreviations: ADHD-RS, attention deficit hyperactivity disorder rating scale; ADHD RS IA, attention deficit hyperactivity disorder rating scale inattentive; ADHD RS HI, attention deficit hyperactivity disorder rating scale hyperactivity index. 
Table 2 ADHD rate of response in 31 bipolar youth at endpoint

\begin{tabular}{|c|c|c|c|}
\hline & $\begin{array}{l}\text { Mania CGI-I } \leq \mathbf{2} \\
\mathbf{N}=\mathbf{2} \mathbf{I}\end{array}$ & $\begin{array}{l}\text { Mania CGI-I > } 2 \\
\mathbf{N}=10\end{array}$ & \\
\hline & $\mathbf{N}(\%)$ & $\mathbf{N}(\%)$ & Statistic \\
\hline \multicolumn{4}{|l|}{ ADHD RS total } \\
\hline $30 \%$ Improvement & $13(62)$ & $2(20)$ & $\chi 2(I)=4.8, p=0.03$ \\
\hline $50 \%$ Improvement & $6(29)$ & $0(0)$ & $\chi 2(I)=3.5, p=0.06$ \\
\hline \multicolumn{4}{|l|}{ ADHD CGI-I } \\
\hline Much Improved & $5(24)$ & $0(0)$ & $\chi 2(I)=3 . I, p=0.08^{*}$ \\
\hline Very Much Improved & $2(10)$ & $0(0)$ & \\
\hline ADHD Responder & $6(29 \%)$ & $0(0 \%)$ & $\chi 2(I)=3.5, p=0.06$ \\
\hline
\end{tabular}

Notes: *Comparison made between CGI-I $\leq 2$ versus $\mathrm{CGI}-\mathrm{I}>2$.

Abbreviations: ADHD-RS, attention deficit hyperactivity disorder rating scale;ADHD-CGI-I, attention deficit hyperactivity disorder-Clinical Global Impressions Improvement Scale.

change from baseline to endpoint in ADHD symptoms was observed only in subjects whose mania responded to risperidone. In these subjects, both inattentive and hyperactive impulsive symptoms significantly improved. Although 62\% of mania responders showed a 30\% reduction in ADHD-RS scores, only $35 \%$ were rated as much or very much improved on the ADHD specific CGI-I ratings and $29 \%$ were rated as responders (CGI $\leq 2$ plus ADHD-RS improvement of $30 \%$ or more).

The most commonly spontaneously reported adverse effects were common cold (39\%), increased appetite (29\%), headache $(20 \%)$, and sedation $(22 \%)$. There were no significant differences between baseline and endpoint in metabolic or cardiac parameters with the exception of a three-fold increase in prolactin levels (from $13.4 \pm 10.1 \mathrm{ng} / \mathrm{dL}$ to $41.3 \pm 23.2 \mathrm{ng} / \mathrm{dL}, \mathrm{F}(1,30)=28.7, \mathrm{p}<0.001)$. In addition there was a statistically significant weight gain over the course of the trial $(2.2 \pm 2.2 \mathrm{~kg} ; \mathrm{F}(1,30)=19.6, \mathrm{p}<0.001)$.

\section{Discussion}

In this prospective, open-label, 8-week treatment trial of risperidone monotherapy in youth with bipolar disorder, we found that treatment with risperidone was associated with statistically significant improvement in symptoms of ADHD that included both symptoms of inattention and hyperactivity. Although improvement in ADHD symptoms was generally modest, these results suggest that risperidone may partially treat symptoms of ADHD in children with bipolar disorder.

These results are consistent with two previous controlled studies of risperidone that documented improvement in ADHD symptoms in children with disruptive behavior disorders and sub average intelligence (Aman et al 2004; Correia Filho et al 2005). The observation that improvement in ADHD symptomatology included both symptoms of inattention and hyperactivity suggests that this improvement was not due simply to the non-specific effects of risperidone on agitation and hyperactivity associated with bipolar disorder.

Our findings documenting that improvement in ADHD symptoms in bipolar children associated with risperidone was limited to those who had an adequate antimanic response are consistent with results from our previous chart review (Biederman et al 1999). That study also documented that the presence of mania interfered with the improvement of ADHD symptomatology during anti-ADHD pharmacotherapy and that ADHD symptoms were much more likely to improve after mood stabilization.

It is noteworthy that despite the observation that $62 \%$ of subjects showed a $30 \%$ reduction in ADHD symptoms, the scores on the ADHD rating scales at endpoint continued to be moderately elevated $(21.2 \pm 10.6)$ indicative of residual ADHD symptomatology. Although more work is needed to evaluate the clinical impact of this level of residual ADHD and whether they warrant additional treatments for ADHD in this population, the partial response to risperidone in ADHD symptomatology needs to be weighed against concerns about the potential destabilizing effects of traditional anti ADHD medications.

Our results need to be viewed in light of methodological limitations. Since this was an open study, the assessments were not blind to treatment. Although firmer conclusions regarding the role of risperidone in the treatment of ADHD symptoms in the context of pediatric bipolar disorder await results from randomized, double-blind, controlled clinical trials, open studies such as this represent an essential first step in developing hypotheses on therapeutic approaches to children with bipolar disorder. Despite these limitations, 
our results suggest that risperidone monotherapy may have modest beneficial effects in controlling ADHD symptoms in youth with bipolar disorder.

\section{Acknowledgments}

This work was supported by grants from Janssen Pharmaceutica (JB), the Stanley Medical Research Institute (JB) and NIH (KO1 MH065523; EM).

\section{References}

Aman MG, Binder C, Turgay A. 2004. Risperidone effects in the presence/absence of psychostimulant medicine in children with ADHD, other disruptive behavior disorders, and subaverage IQ. J Child Adolesc Psychopharmacol, 14:243-54.

Ambrosini PJ. 2000. Historical development and present status of the schedule for affective disorders and schizophrenia for school-age children (K-SADS). J Am Acad Child Adolesc Psychiatry, 39:49-58.

Biederman J, Faraone S, Wozniak J, et al. 2004. Further evidence of unique developmental phenotypic correlates of pediatric bipolar disorder: Findings from a large sample of clinically referred preadolescent children assessed over the last 7 years. $J$ Affect Disord, 82:S45-S58.

Biederman J, Mick E, Faraone SV, et al. 2000. Pediatric Mania: A developmental subtype of bipolar disorder? Biol Psychiatry, 48:458-66.

Biederman J, Mick E, Hammerness P, et al. 2005a. Open-label, 8-week trial of olanzapine and risperidone for the treatment of bipolar disorder in preschool-aged children. Biol Psychiatry, 58:589-94.

Biederman J, Mick E, Prince J, et al. 1999. Systematic chart review of the pharmacologic treatment of comorbid attention deficit hyperactivity disorder in youth with bipolar disorder. J Child Adolesc Psychopharmacol, 9:247-56.

Biederman J, Mick E, Wozniak J, et al. 2005b. An open-label trial of risperidone in children and adolescents with bipolar disorder. $J$ Child Adolesc Psychopharmacol, 15:311-17.
Borchardt CM, Bernstein GA. 1995. Comorbid disorders in hospitalized bipolar adolescents compared with unipolar depressed adolescents. Child Psychiatry Hum Dev, 26:11-18.

Correia Filho AG, Bodanese R, Silva TL, et al. 2005. Comparison of risperidone and methylphenidate for reducing ADHD symptoms in children and adolescents with moderate mental retardation. $J$ Am Acad Child Adolesc Psychiatry, 44:748-55.

Eapen V, Gururaj AK. 2005. Risperidone treatment in 12 children with developmental disorders and attention-deficit/hyperactivity disorder. Prim Care Companion J Clin Psychiatry, 7:221-4.

Faedda G, Baldessarini R, Suppes T, et al. 1995. Pediatric-onset bipolar disorder: A neglected clinical and public health problem. Harv Rev Psychiatry, 3:171-95.

Geller B, Craney JL, Bolhofner K, et al. 2002. Two-year prospective followup of children with a prepubertal and early adolescent bipolar disorder phenotype. Am J Psychiatry, 159:927-33.

Geller B, Luby J. 1997. Child and adolescent bipolar disorder: A review of the past 10 years. $J$ Am Acad Child Adolesc Psychiatry, 36:1168-76.

Geller B, Sun K, Zimmerman B, et al. 1995. Complex and rapid-cycling in bipolar children and adolescents: A preliminary study. $J$ Affect Disord, 34:259-68.

National Institute of Mental Health. 1985. CGI (Clinical Global Impression) Scale - NIMH. Psychopharmacol Bull, 21:839-44.

Strober M, Schmidt-Lackner S, Freeman R, et al. 1995. Recovery and relapse in adolescents with bipolar affective illness: A five-year naturalistic, prospective follow-up. J Am Acad Child Adolesc Psychiatry, 34:724-31.

West S, McElroy S, Strakowski S, et al. 1995. Attention deficit hyperactivity disorder in adolescent mania. Am J Psychiatry, 152:271-73.

Wozniak J, Biederman J, Kiely K, et al. 1995. Mania-like symptoms suggestive of childhood onset bipolar disorder in clinically referred children. $J$ Am Acad Child Adolesc Psychiatry, 34:867-76.

Young R, Biggs J, Ziegler V, et al. 1978. A rating scale for mania: Reliability, validity and sensitvity. Br J Psychiatry, 133:429-35.

Youngstrom EA, Danielson CK, Findling RL, et al. 2002. Factor structure of the Young Mania Rating Scale for use with youths ages 5 to 17 years. J Clin Child Adolesc Psychol, 31:567-72. 
